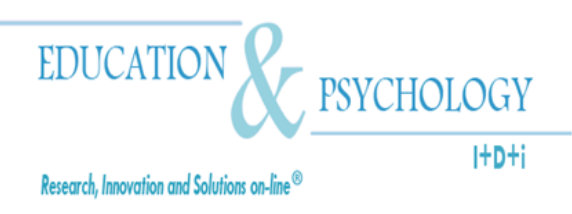

\title{
Implicaciones de la Formación en la Autonomía del Estudiante Universitario
}

\section{Catherina De Luca}

Secretaria Ejecutiva del Rectorado, Universidad Nacional Experimental de los Llanos Occidentales Ezequiel Zamora, Barinas

\section{Venezuela}

Catherina De Luca. Urb. Fundación Mendoza, Casa 16-69, Barinas, Estado Barinas. Código Postal: 5201. Venezuela. E-mail: opeiunellez@gmail.com

(C) Education \& Psychology I+D+I and Editorial EOS (Spain) 


\section{Resumen}

En el trabajo que se presenta se configuran algunas líneas teóricas que definen la autonomía en el aprendizaje desde la perspectiva de los principales conceptos y teorías que se debaten actualmente. Su contenido se estructura en tres dimensiones de análisis. En la primera dimensión se presenta una aproximación al concepto de autonomía, y se establece una discusión que trata de clarificar la relación entre teoría y práctica del autoaprendizaje significativo. En la segunda dimensión, se aborda las implicaciones de la autonomía y la heteronomía en el aprendizaje y, finalmente, en la tercera dimensión se expresan las conclusiones para el abordaje de una posible intervención.

Palabras clave: implicaciones, autonomía, formador, estudiante, heteronomía 


\title{
The implications of educational preparation in the university student's autonomy
}

\begin{abstract}
In the present paper some theoretical lines are configured which define the autonomy in the learning from the perspective of the main concepts, models and theories that it is debated at the moment. Their content is structured in three analysis dimensions, in the first dimension an approach is presented to the concept of autonomy, and a discussion that tries to clarify the relationship between theory and practice of the significant autoaprendizaje settles down. In the second dimension it is approached the implications of the autonomy and the heteronomy in the learning and, finally, in the third dimension the summations and recommendations are expressed for the boarding of a possible intervention.
\end{abstract}

Keywords: implications, autonomy, formador, student, heteronomy.

Received: 01/16/09

Initial Acceptance: 02/04/09

Final Acceptance: 02/24/09 


\section{Introducción}

Se trata de asumir la discusión entorno al concepto de autonomía que maneja el profesorado en su rol como formador de formadores, de allí que, en la reflexión teórica que se plantea en los apartados del estudio se parte por definir desde el punto de vista sistémico la autonomía como la capacidad de darse a sí mismo normas, con vistas a la praxis, y asumir la propia vida en función de dicha posición.

La afirmación del ser humano como autonomía absoluta, de raigambre kantiana, entiende al hombre capaz de darse a sí mismo las normas de conducta como un verdadero legislador universal, porque entiende que no existe nada en este mundo que resulte tan universal como la conciencia libre para actuar y hacer, configurándose las leyes de su propio accionar individual y social.

En concordancia con la capacidad de obrar libremente, Berner (1988) manifiesta que la razón pura práctica es fundamentalmente autolegisladora, es decir, autónoma (del griego autós: sí mismo; y nómos: ley), y si, cuando se habla de este asunto, es inevitable acordarse de Kant, probablemente el padre de la autonomía a ultranza, del formalismo ético y del deber por el deber, entonces resulta obligado citar al menos unas pocas pero muy clásicas afirmaciones kantianas: dos cosas llenan el ánimo de admiración y de respeto, siempre nuevas y crecientes, cuanto con más frecuencia y aplicación se ocupa de ellas la reflexión: el cielo estrellado sobre mí y la ley moral en mí.

\section{Aproximación al concepto de autonomía}

Existe una diversidad de definiciones acerca de la autonomía, cada una de ella ha estado matizada por el concepto que el autor ha manejado de ella, sin embargo, existen algunas que están más centradas en la persona, en dejar clara la esencia y voluntad humana en el proceso de construcción de la libertad para actuar, decidir y transformar. En este orden de ideas se revisan algunos autores:

La autonomía es entendida como la libertad que posee un individuo para disponer de sí mismo, expresar sus sentimientos, ideas y emociones, sin limitaciones. De acuerdo con la enciclopedia Larousse (1995), la autonomía es la capacidad que tiene el individuo de partici- 
par en la construcción de acuerdos a través de la mediación y el intercambio de puntos de vistas con las demás personas.

Para Riso (2004) "el principio de autonomía te enseña a ser independiente. A verte a ti mismo sin convertirse en antisocial, te muestra el camino de la emancipación psicológica y afectiva. Cuando una persona decide tomar las riendas de tu vida, los apegos no prosperan fácilmente". La autonomía, más específicamente, se describe como la capacidad de la persona para autodirigirse; es decir, la capacidad de gobernarse a sí mismo, con base en un código de conducta asumido personalmente, teniendo en cuenta la relación constitutiva con los otros.

El fin último de la educación es formar hombres y mujeres que sean libres para aprender. La universidad, consciente de esta dimensión, espera que sus estudiantes tengan una formación sólida, que los capacite para tomar decisiones conscientemente fundadas e inspiradas en los valores fundamentales que han adquirido en su desarrollo autónomo como sujetos.

Para Rubio (1992), la autonomía es uno de los valores morales en general, no es una capacidad individual, no es un estado o atributo de las personas, sino un ejercicio, una cualidad de la vida que viven. Se tiene que hablar, por tanto, de procesos y situaciones sociales en las que las personas se conducen automáticamente y, en ese proceso, construyen su identidad ética.

La palabra autonomía implica la ruptura de la heteronomía. Los límites de la autonomía individual están dados porque ésta nunca es posible si no coincide con la autonomía del conjunto. El sentido último de la autonomía es darse la propia ley; pero, se trata de una ley como auto creación de la sociedad, que no reconoce fundamentos extra sociales. La autonomía se desarrolla en un contexto de relaciones, dentro de la sociedad; tiene que ver, por tanto, con una forma de mantener relaciones y son, por consiguiente, las cualidades que reúnan éstas las que puedan favorecer o entorpecer. Sólo en el deseo de que los alumnos asuman protagonismo en su vida escolar y en su aprendizaje puede entenderse la preocupación de un profesor por entablar un diálogo o buscar el entendimiento mutuo. Sólo bajo una concepción no impositiva de la enseñanza puede entenderse la aspiración a una autonomía que se construye en la relación. 
Finalmente, consideramos que la autonomía es la capacidad que tiene el sujeto, en nuestro caso, el estudiante universitario que se prepara para formar en el magisterio venezolano, de expresar libremente sus pensamientos, ideas, emociones y asumir posición frente a una situación determinada dentro del aula de clase, a pesar de que sus puntos de vista, sean distintos al de su profesor.

\section{Características de la autonomía}

De acuerdo con Alanis (2003), la autonomía es un ideal moderno que ha funcionado como uno de los horizontes de sentido más significativo de la humanidad. Es, en sí misma, la autonomía, el criterio moral por excelencia o el indicador real de la libertad. La autonomía es construida, merecida y ejercida. Es un valor construido, en cuanto, no aparece ante el individuo como un hecho dado o inherente a su condición humana, sino que es el resultado de un proceso de crecimiento que requiere del sujeto un esfuerzo consciente que se gesta a lo largo de la vida y en donde intervienen aspectos evolutivos de carácter cognitivo y moral que en últimas le permita hacer el transito de la situación de heteronomía hacia la mayoría de edad o la autodeterminación.

Es la autonomía, un merecimiento, por cuanto poseerla es el premio a un esfuerzo personal desde donde se programa el transito de la obediencia ciega a normas propuestas desde el exterior del individuo hacia la obediencia consciente y voluntaria a principios libremente elegidos y es a la vez un derecho, puesto que nadie puede privarnos del pensar y decidir por sí mismos.

Es un ejercicio, toda vez que ella existe sólo en la capacidad para elegir, decidir, justificar y racionalizar las acciones humanas. La autonomía es entonces el ejercicio de libertad y el fundamento de la dignidad humana, pues el ser humano se destaca de las demás criaturas gracias al ejercicio de su racionalidad y en consecuencia la capacidad de darse la propia ley. 


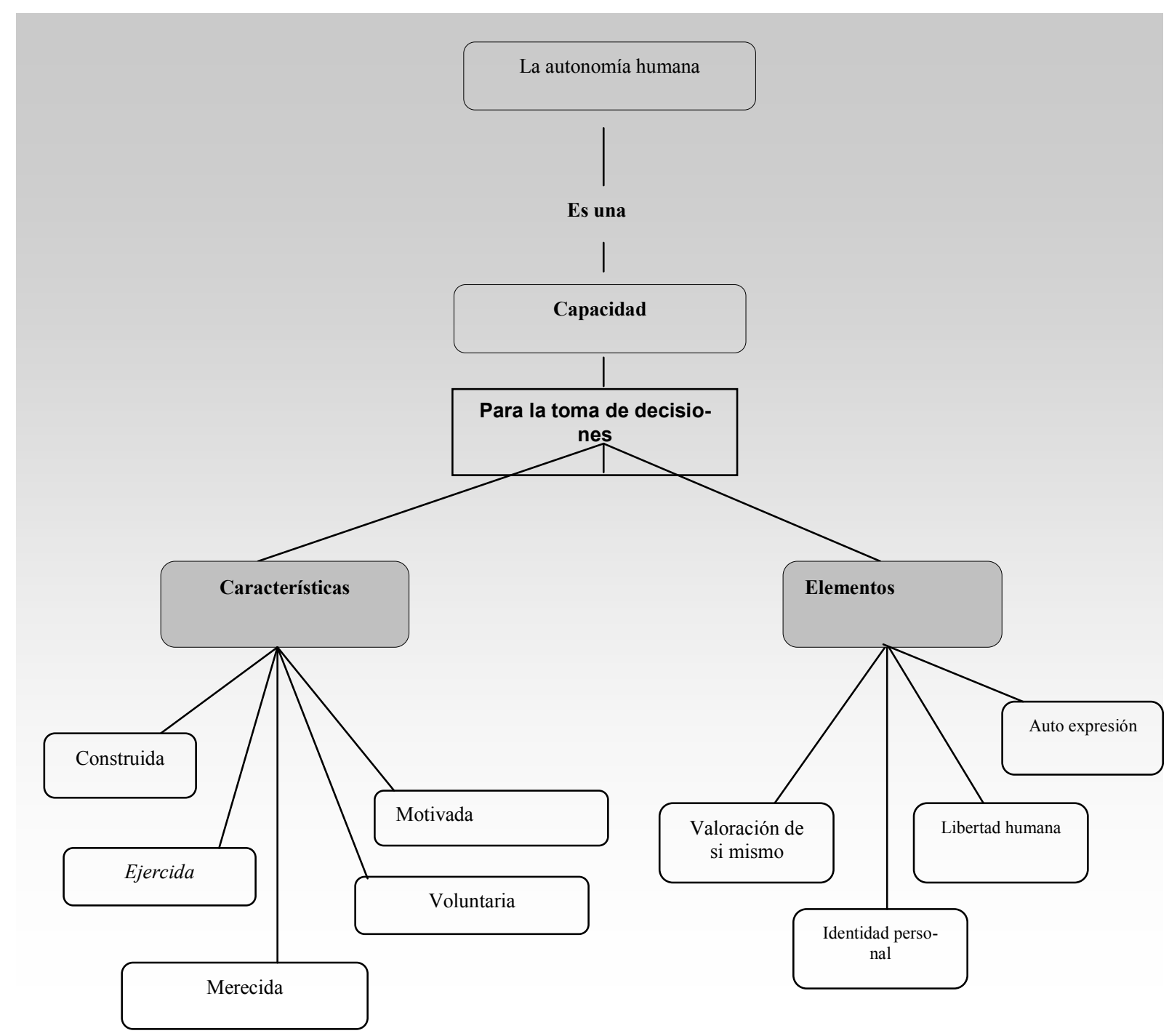

Fuente: De Luca (2008)

Figura 1. Elementos implicados de la autonomía humana

Lo anterior permite afirmar que la autonomía es la capacidad de cada hombre y mujer para gobernarse a sí mismo, donde el primer requisito de una conducta autónoma lo constituye la decisión, por parte del ser humano de asumir directamente la responsabilidad de su propia vida y de no dejar que otros decidan en su lugar. Es la autonomía el producto de una voluntad que decide encausar su vida de acuerdo con patrones propios, es tener un proyecto y ajustar las acciones a los fines propuestos, lo cual implica a su vez una especie de autocontrol o disciplina indispensable para canalizar las energías vitales hacia el logro de los fines libremente escogidos. 
La autonomía es una construcción permanente, es una constante activación de lo que somos como seres individuales y sociales, que luchamos constantemente por el flujo de energía y factores tanto internos como externos que fortalecen a la persona con bases sólidas, pero que incidir en otras con menos soporte moral y ético.

El ejercicio de la autonomía exige así, en la persona la capacidad y la voluntad de tomar las riendas de su propia vida, organizarla con criterios de racionalización pragmática y de racionalidad moral, y tratar de cultivar una individualidad ricamente desarrollada y relativamente original.

Tener derecho a la autonomía no significa elegir al antojo, significa que para elegir no sólo nos guiamos por el tribunal de nuestras conciencias, sino que es necesario asumir en lo posible una perspectiva imparcial de juicio y reconocer la igual libertad de los demás. El derecho de la autonomía supone por igual la disponibilidad de escuchar y aprender de los demás, pues comunicarse es aprender a trabajar por la capacidad de autodirección racional.

Lograr la mayoría de edad no nos libera de la obligación de confrontar nuestros puntos de vista con las opiniones de los demás. Sólo desde el diálogo podemos hacer uso de nuestra libertad de pensamiento y de conciencia, es decir, no somos solos, ni somos libres, sino que nos hacemos libres en el contacto y la exposición de nuestros pensamientos ante los demás.

Teniendo en cuenta lo expuesto hasta el momento podríamos entonces afirmar que la autonomía es producto de un proceso de desarrollo individual y social, construido al interior de los colectivos pasando por la familia, la escuela, el liceo y la universidad, e incluso a lo largo de toda la vida de la persona.

Los elementos que describen la autonomía son diversos. En la figura 2, se han agrupado los aspectos personales y sociales que se potencian para promover la autonomía del sujeto que aprende. Desde el humanísimo, la autonomía esta vinculada la cultura, a la dinámica social que hace que el ser humano integre nuevos valores y asuma nuevas actitudes que se van construyendo con la experiencia. 


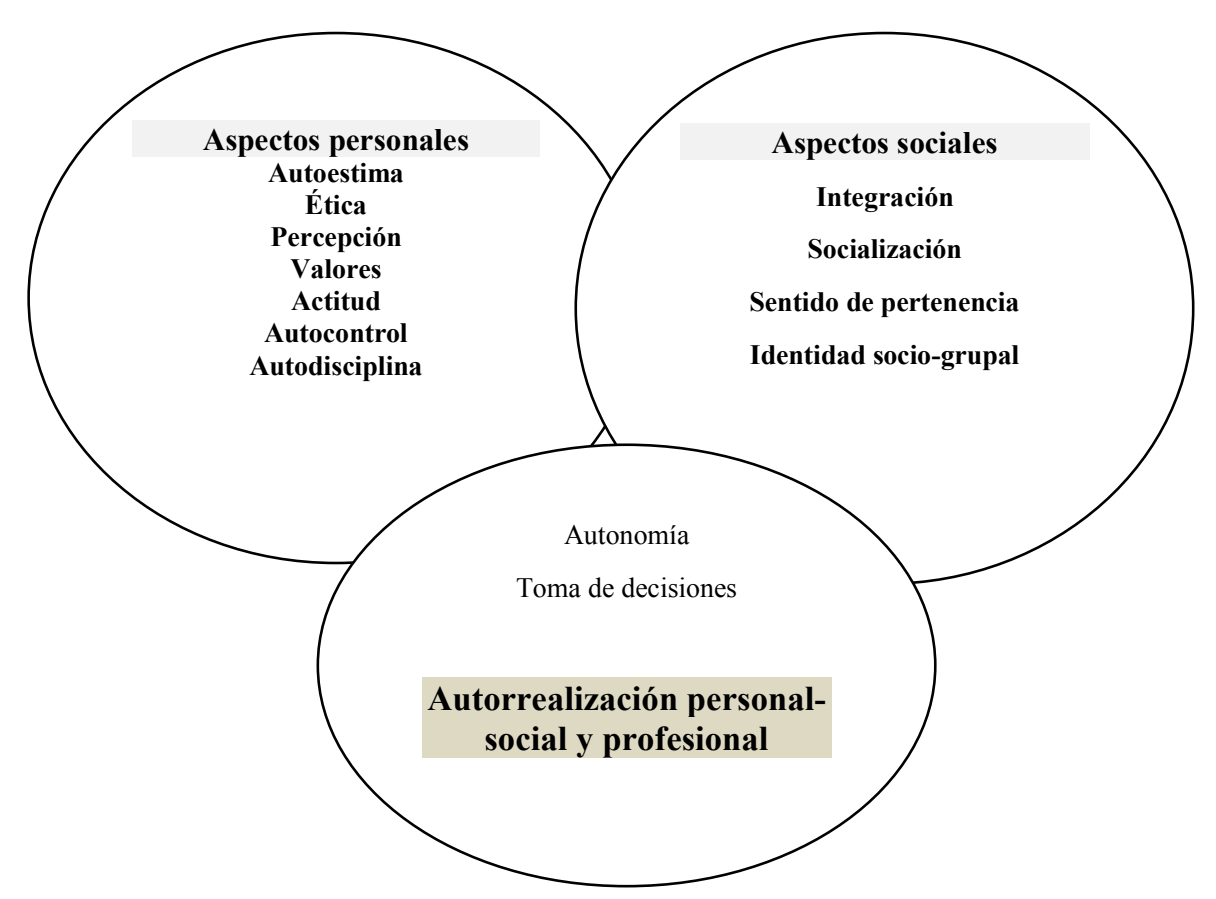

Fuente: De Luca (2008)

Figura 2. Aspectos personales y sociales potenciadores de la autonomía

En la medida en que nos evaluamos y conocemos, nos acercamos más al autoconocimiento, percatándonos de la libertad que disponemos o de la capacidad que poseemos para tomar decisiones libres, para dialogar e intercambiar ideas, con un estilo propio, maximizando el potencial analítico y creativo con el que contamos, fortaleciendo los valores y principios sociales a los que responde el comportamiento.

Realizadas las consideraciones anteriores, pensamos que hay razones para estudiar la autonomía ya que en la medida en que se promueva más tempranamente en el desarrollo de las personas, se tendrán mayores posibilidades de construir una cultura de convivencia, donde el respeto por las normas y por los demás permita la posibilidad de crear espacios para intercambiar maneras de pensar y hacer frente a los problemas a pesar de las actitudes que prevalezcan.

Fomentar la autonomía de los estudiantes es un tema controversial y a juicio de muchos docentes, de gran importancia tanto en el ámbito educativo básico como en el superior. Se reconoce o al menos está escrito en reglamentaciones las normativas de la educación venezolana, la necesidad de formar sujetos críticos, reflexivos y con capacidad de formarse sus 
propios juicios. Y es que ciertamente, la característica sobresaliente del ser humano es precisamente la posibilidad de tener no sólo pensamiento, sino poder reflexionar sobre él y actuar en los procesos de transformación social para su beneficio y del colectivo.

En efecto, la autonomía es la meta hacia dónde se dirigen los esfuerzos de los educadores y educandos, para que esta capacidad del alumno pueda ser estimulada y fomentarse en él, que su pensamiento subjetivo, al verse enfrentado o contrapuesto con el pensamiento subjetivo de los otros seres humanos que le rodean, pueda ser conocido y juzgado, buscando un equilibrio tanto emocional como intelectual.

Son diversos los factores que inciden en el complejo proceso de la enseñanza. Por ejemplo, a las estrategias de enseñanza se les ha dado gran importancia para el logro de los diferentes objetivos que se plantea un profesor en el proceso antes mencionado. Se tiene el convencimiento de que exclusivamente los conocimientos impartidos por el profesor no son los que deberían privar en dicha relación. Se escucha con frecuencia la necesidad de formar profesionales con elevado nivel de preparación capaces de competir y enfrentarse a un mundo globalizado.

En este orden de ideas, la educación debe ir más allá de esa importante misión y lograr el desarrollo autónomo de los individuos, que los capacite para enfrentar los difíciles retos que se les plantean en la vida diaria. Siendo capaces de asumir responsabilidades y posturas firmes ante los diferentes problemas que deban enfrentar. En el caso particular de los estudiantes que se forman para convertirse en docentes a futuro se observa con preocupación cómo la pasividad, sumisión y poca participación han tomado las aulas de clase donde estos jóvenes se forman, aun siendo ellos quienes se preparan para formar a los niños que, en el futuro, representarán al país en los diversos ámbitos del acontecer nacional.

Es fundamental la presencia de la autonomía, y la búsqueda de valores a través de acciones humanistas (ser coherentes, fieles a nosotros mismos, veraces, auténticos, solidarios). Se piensa que una manera para fomentar la autonomía del estudiante, podría ser a través de las estrategias utilizadas por el docente. No obstante, se observa que el papel desempeñado por los formadores de formadores está centrado en cumplir con un currículo programado, sin conocer si se están logrando o no las expectativas del perfil del egresado, en el que deben estar 
presentes características personales contempladas en el normativo de la Educación como la creatividad, criticidad, la participación y la autonomía, entre otras.

\section{Disonancia entre teoría y práctica de la autonomía}

El estudio de las contradicciones entre la autonomía humana y la práctica de la autonomía en el proceso formativo, lleva a revisar necesariamente lo que plantea Festinger acerca de la teoría de la Disonancia Cognitiva, citado por Vadillo (2004, p.17) "las personas no soportamos mantener al mismo tiempo dos pensamientos o creencias contradictorias, y automáticamente, justificamos dicha contradicción, aunque para ello sea necesario recurrir a argumentaciones absurdas", es decir, el ser humano necesita siempre sentir que todas sus acciones, pensamientos y creencias son coherentes. En consecuencia los conceptos que a lo largo de toda la vida hemos venido construyendo y reforzando con los pensamientos incongruentes se traducen en actos y prácticas formativas contradictorias en nuestro deber como formadores y para reducirlas recurrimos a la "Justificación Insuficiente" o "Autojustificación".

En efecto algunos profesores durante la práctica docente universitaria, con estilos de enseñanza autocráticos, poco creativos y con rituales clásicos y tradicionales para impartir las asignaturas, justifican así la limitadas oportunidades que les brindan a sus estudiantes para el desarrollo de la autonomía humana: ¿cómo es posible que hayan alumnos con una enorme dificultad para expresarse libremente y para mantener sus puntos de vista, por eso me corresponde conducir todas las actividades de aprendizaje y darles todas las orientaciones para que puedan producir algo?, $\measuredangle$ alumnos que dependen de las opiniones de los otros, eso ha hecho que les asigne mayor responsabilidad a ellos, los demás integrantes del grupo no son responsables?, ¿no entiendo por qué los estudiantes no han logrado seguridad y les he dado todo en el aula?, ¿qué hacen para desarrollar esta habilidad o valor?, ¿y por qué algunas personas nos preocupamos y comentamos estas situaciones cuando observamos lo que ocurre con frecuencia en las aulas de clase?

Estas interrogantes muestran la necesidad que tenemos las personas de justificar lo innecesario o, incluso, lo injustificable. Lo realmente importante para nosotros es creer que no existen contradicciones entre varios de nuestros pensamientos y creencias, y no sólo necesitamos convencer a los demás de la coherencia de nuestros actos, sino también a nosotros 
mismos. Por tanto, no actuamos por alguna razón, sino que actuamos y luego buscamos razones que expliquen nuestra actuación.

Lo anteriormente descrito, se verifica en situaciones muy diversas en las aulas de clases: no participando, mostrando una actitud sumisa, haciendo críticas sin aportar soluciones, en la escasa creatividad mostrada, en la baja productividad, poca cooperación, en el temor de hablar públicamente. También se verifica, en la limitada iniciativa en la gestión personal. En este sentido, los alumnos toman decisiones que muchas veces no obedecen a sus propias convicciones, por el contrario, se sujetan a lo que otros creen que debe decidirse, generando la disonancia entre el deber ser reflexionado y la práctica del ser distorsionado.

Estas características y rasgos evidentes en el individuo se han visto reforzados por esquemas y modelos asumidos por el profesorado para impartir la enseñanza en las aulas, y por consiguiente ha dirigido a los estudiantes hacia procesos de aprendizaje más dependientes. Estableciéndose de esta manera, una relación de poder y de subordinación durante el acto de enseñar y aprender. Además de la actitud del profesor, se observan prácticas asociadas y dirigidas con mayor énfasis al dominio de conocimientos y escasa promoción del desarrollo de habilidades y destrezas que están en sintonía con los esquemas de la educación tradicional, al concebir al sujeto como un ser reproductivo y obediente.

La negación de la libertad natural del sujeto, de la trascendencia de los aportes que pueda hacer en los grupos sociales y escolares. Las limitadas oportunidades para la construcción de nuevos aprendizajes, de nuevas formas de abordar los problemas. El restringido espacio para la discusión y la escasa atención que se da al desarrollo personal de los estudiantes, entendemos que sostiene actitudes en los mismos que le refuerzan y acentúan lo que hemos considerado la antítesis de lo que debe ser un estudiante con autonomía.

La autonomía es un tema que ha despertado la curiosidad de los psicólogos y docentes; sin embargo es escasa la información que se encuentra sobre este valor. Sin embargo, desde una perspectiva histórica, la mayor parte de investigaciones y de intentos en dar formación docente en el ámbito de la educación, y el trabajo autónomo está orientado en la concepción de la enseñanza eficaz o eficacia docente. 
Al respecto, Díaz y Hernández (2007), coinciden en que los principales problemas, tanto teóricos como metodológicos que han enfrentado en la formación de maestros autónomos son: presuponer una estabilidad de rasgos de personalidad con independencia del contexto donde se manifiestan; multidimensionalidad de los métodos de enseñanza y las dificultades en su operacionalización; así como la falta de control de variables en el escenario natural.

Así mismo, se analiza como un problema apremiante en la formación de los formadores la forma teórica y poco favorecedora del desarrollo de la autonomía que se promueve desde el uso de las estrategias metodológicas utilizadas en el proceso formativo. Frente a ello Rodríguez (2007) señala que durante el aprendizaje autónomo el participante debe reconocer la importancia que tiene para el tener su propio programa de aprendizaje; en este no solo el facilitador o docente es quien debe programar. Pero la autonomía de aprendizaje no debe ser total más bien debe ser parcial, simplemente se establecen estrategias según las características personales de cada estudiante para que el desarrolle un elevado nivel de responsabilidad de su formación y su evaluación.

Para Serrano (2002), la propuesta del estudiante-profesor autónomo y reflexivo se sujeta en una coherencia práctica, donde la formación de los profesionales enfatiza la acción práctica, por razón de la compresión plena de la situación donde se labora, la cual sólo puede alcanzarse por la vía de procesos de deliberación, debate e interpretación. El papel del docente no es en este caso el de un operario o técnico que aplica sin más los planes, programas o metodologías pensadas por otros, sino que se convierte en un profesional reflexivo que rescata su autonomía intelectual.

Sobre la base de las consideraciones anteriores, los aportes que el docente puede dar a la problemática que enfrenta en el aula y en la institución académica en general, dependerán de la construcción que haga de situaciones donde suele prevalecer la inseguridad, la singularidad y el conflicto de valores. Entre otras, una de las alternativas propuestas es la experiencia de aprender haciendo y el arte de una buena acción tutorial, ello implica la necesidad de reflexión de la práctica de lo que para el estudiante de magisterio será el futuro.

La formación de un docente autónomo se estructura alrededor de unas prácticas que aspiran ayudar a los estudiantes a adquirir las formas de "arte" que resultan esenciales a su competencia académica, personal o profesional. Sobre este aspecto, los talleres de trabajo y 
las actividades libres en escenarios naturales, la práctica de una buena tutoría, así como las diferentes formas de interacción entre estudiantes y docentes constituyen las piezas clave de la propuesta para la formación de un sujeto autónomo.

Experiencias como la de Matheus (2004) plantea los siguientes interrogantes: ¿por qué los profesores hoy queremos que nuestros estudiantes universitarios se emancipen de nosotros?, ¿quién requiere un futuro profesional dispuesto y capaz de hacerse cargo de sus propios procesos de aprendizaje. Para este autor, exigir una autonomía del estudiante, en su caso del estudiante de idiomas extranjeros, es más que un intento del docente de buscar formas más eficaces de aprender, para que se obtengan resultados más satisfactorios y mejores notas. Exigir autonomía es una necesidad que surge de la sociedad en general y del mercado laboral en particular. Ambos exigen de las personas máxima flexibilidad y capacidad de adaptación y el desarrollo de habilidades sociales y afectivas que le permiten establecer relaciones productivas y proactivas. La idea se puede ilustrar en el caso de un estudiante que se enfrenta con la dificultad de aprender un idioma. La globalización o internacionalización ya no es una frase sólo del mundo económico, sino también de la enseñanza superior en donde muchos alumnos y profesores están participando.

¿Cómo puede ser la visión del "estudiante autónomo" en una institución de enseñanza superior?, ¿cuánta autonomía es posible allí, qué cantidad es la deseable? Como dice Riso (2004), el concepto de "autonomía" implica también "libertad", la que a veces es interpretada como la "libertad del control del profesor", "la libertad del currículo", incluso como la "libertad de tomar decisión de no aprender". Esta noción de "autonomía" no será compatible en la mayoría de los casos con la realidad de las universidades donde rigen planes de estudio y donde los reglamentos, incluso pueden exigir la asistencia del alumno a clase. Pero, aunque los contenidos y los objetivos de la enseñanza de idiomas en el contexto institucional sean relativamente fijos, hay muchos caminos válidos para alcanzar éstos últimos. En este contexto, al estudiante independiente lo podemos definir como una persona:

- Que conoce los objetivos de su aprendizaje.

- Que tiene plena conciencia de sus puntos fuertes y débiles.

- Con capacidad de reflexión y auto evaluación.

- Que se considera como el centro de su aprendizaje y ve en el profesor (sólo) a un orientador y consultor competente. 
- Que ha adquirido conocimientos sólidos sobre técnicas de aprendizaje experimentando con ellas, de manera que es capaz de escoger las técnicas adecuadas para elaborar una estrategia individual, que le permite enfrentar en forma flexible las tareas que le son presentadas por otros (profesores) o por él mismo.

Estas tareas las resuelve manejando técnicas que van desde la auto motivación y la planificación del proceso de aprendizaje hasta las micro técnicas para el uso de fuentes de información, para aprender vocabulario y gramática, técnicas de lectura etc., según sea su necesidad.

Pero el alumno autónomo también se está preparando, para un futuro rol de autodidacta, dispuesto y capaz de aprender o perfeccionar un oficio por cuenta propia. Esto puede significar que la persona en el futuro estudie un idioma vía Internet, haciendo uso de la educación a distancia que en este momento está adquiriendo una notable importancia. La formación de este tipo de estudiante es una gran meta, pero al mismo tiempo hay que considerarlo como un medio para alcanzar el objetivo principal de la enseñanza.

De acuerdo con Pérez (1994) los aprendices aprenden sólo cuando encuentran sentido a lo que aprenden. De lo contrario no sólo no aprenden, sino que además molestan y se convierten en deprimidos socioculturales, porque carecen de las herramientas para tener acceso a su propia cultura." (...) "Partir de la experiencia previa. Esto supone que la arquitectura del conocimiento se apoya en el suelo, y el suelo de lo que se aprende tiene dos supuestos: lo que el alumno sabe y lo que sabe hacer con lo que sabe, su propia experiencia".

Estos dos supuestos, son los que nos llevaron a plantear y discutir en grupos pedagógicos con los docentes; por un lado, para enriquecer el saber, construirlo en el intercambio y por otro, para enseñar desde la propia experiencia. Creemos en la necesidad de una autonomía creciente en desarrollo del proceso de aprendizaje de los estudiantes de la Carrera de Educación. Capacidad demostrada para autoevaluar su propio desempeño. Autodisciplina en su desenvolvimiento institucional, capacidad y necesidad de socializar los saberes adquiridos. 


\section{Concepción de sí mismo vs concepción del otro: heteronomía}

Tratar de entender la heteronomía, pasa por una revisión de la obra El Si-mismo como otro de P. Ricoeur, citado por Masial (2002), que se centra en la pregunta « ¿quién?»: ¿quién habla>, ¿quién actúa?, ¿quién es el sujeto de la narración?, ¿quién es el sujeto de la imputación?, tratando de evitar tanto la exaltación cartesiana como la humillación nietzscheana del sujeto, da Ricoeur el rodeo hermenéutico de pasar por las teorías del lenguaje, de la acción, de la narratividad y de la ética. El «Sí-mismo », más que un punto de partida, resulta ser un punto de llegada tras el rodeo de sus expresiones. Este esfuerzo de recuperación del sujeto y su autonomía pasando por el otro puede concebirse como una revisión de la heteronomía.

Hay una estructura dual de la conciencia voz que llama y voz que responde desplegada en forma de estructura dialogal de la vida humana. Hay una alteridad constitutiva del «Símismo » (al que no se llama «yo» porque decir «Sol» es más que decir. En relación dialéctica con esa alteridad se constituye la ipseidad. El Sí-mismo y el otro se implican mutuamente. Cada uno de ellos son un yo, un tú y un él, ella, vinculados correlativamente para ser autónomos. Tal concepción de sí mismo a la luz del otro supone una revisión de la heteronomía, así como de la misma autonomía.

La heteronomía.- significa que las acciones son creadas por otra persona distinta al destinatario de la acción, y, que ésta, además, es impuesta en contra de su voluntad; esta característica se opone a la autonomía que significa que la acción es creada de acuerdo a la propia conciencia de la persona, es auto legislación (darse sus propias leyes).

Se denomina heteronomía a la voluntad no determinada por la razón del sujeto, sino por algo ajeno a ella (la voluntad de otras personas, las cosas del mundo, la sensibilidad, la voluntad divina, etc.)

La heteronomía de la voluntad escribe la circunstancia de que cuando un sujeto sigue leyes morales; las leyes a las que está sometido no tienen origen en su propia razón sino que le vienen dadas de fuera. La voluntad puede estar determinada por dos principios, puede tener dos fundamentos: la razón o la inclinación. Cuando es la propia razón la que decreta el modo en que debe actuar la voluntad, ésta es autónoma porque se da a sí misma sus propias leyes. 
Sin embargo, cuando la voluntad está determinada por la inclinación (palabra con la que Kant se refiere al conjunto de apetitos sensibles) la voluntad es heterónoma.

Reiterando lo mencionado en párrafos anteriores existen diversos indicadores que muestran la heteronomía intelectual de los estudiantes, observados a lo largo de mis años de experiencia docente, entre los cuales pueden citarse los siguientes:

- Temor a manifestar sus puntos de vista.

- Inseguridad al hacer planteamientos.

- Permanecer en silencio.

- Insistencia en recibir del profesor una calificación mientras realiza una actividad.

- Búsqueda continúa de aprobación por parte de profesor.

Ante la situación mencionada, se hace necesario generar acciones que permitan al estudiante encontrar las respuestas a sus propias preguntas, por medio de hechos concretos, que lo lleven a ser capaz de pensar por sí mismo con sentido crítico, teniendo en cuenta muchos puntos de vista, tanto en el ámbito moral como en el intelectual; dado que existe una necesidad urgente de participación y desarrollo de la autonomía que contribuya a enfrentar aspectos sociales y educativos del país.

Se sabe que no es tarea fácil; sin embargo, se parte de la necesidad de formar profesionales con una condición intelectual que no les haga depender de nadie, con capacidad para encontrar las respuestas a sus propias preguntas, con libertad para tomar sus propias decisiones y, en este sentido, se hace necesario tomar en consideración aspectos planteados por la teoría constructivista al enfatizar lo importante que es desarrollar potencialidades innatas en el sujeto que construye sus aprendizajes, sin olvidar la posibilidad de utilizar estrategias que, empleadas por el docente, faciliten en el alumno la activación de procesos mentales que reafirmen su autonomía y minimicen la heteronomía con que acomete sus aprendizajes.

El desarrollo de la autonomía en estudiantes de nuestra cultura no es sencillo. En este sentido, Freire (1997) se interesó particularmente en el desarrollo de la formación permanente de los profesores, con el fin de que este proceso permita la reflexión crítica sobre la práctica, considerando que pensando críticamente la práctica de hoy, o la de ayer, es como puede mejorar la próxima. 
Investigaciones centradas en el aprendizaje por descubrimiento, tienden a fomentar el trabajo autónomo, tomando en consideración una triple direccionalidad: adaptación, iniciativa y responsabilidad.

De esta manera, tomar en cuenta la adaptación significa contextualizar la enseñanza a las necesidades personales de cada estudiante (intereses, capacidades, ritmo de aprendizaje y otros). Considerar la iniciativa implica capacitar a los estudiantes en la selección y manejo de materiales adecuados que satisfagan sus necesidades educativas y tender a la responsabilidad sería contribuir a desarrollar la capacidad de respuesta respecto a la utilización de los medios que la escuela y el maestro ponen a su disposición, asumiendo que los éxitos son suyos y los fracasos también.

Desarrollar y fomentar la autonomía del estudiante en el contexto venezolano implica educar al alumnado y profesorado en la implementación de un método didáctico innovador, creativo, cooperativo ajustado a las necesidades de los alumnos, donde la conducta esté centrada en el trabajo independiente del profesor, mostrando iniciativa hacia la investigación creativa, innovadora, generadora de conocimientos.

Esta práctica requiere del compromiso de ambas partes (profesorado y alumnado) y de una tarea de instrumentación previa, técnicas de trabajo que unidas al potencial intelectual y creativo de ambos beneficie el trabajo autónomo en el aula y fuera de ella.

Rodríguez (1995) se refiere en una de sus obras, donde diserta sobre las relaciones entre la educación para la paz y el interculturalismo como tema transversal, al sentido que tiene educar a los alumnos desde los temas transversales, llevando consigo las siguientes implicaciones a juicio: -ayudar a los estudiantes a tomar decisiones, tarea nada fácil, según hemos venido analizando, el mundo en que viven esos educandos, es un mundo desenfrenado, plagado de problemas y conflictos, donde la fragmentación oculta lo esencial, donde se ha manifestado el ocaso de los mitos que en otro tiempo alumbraban el sendero, donde se ha operado la inversión de los valores, donde según otra vez Nietzsche solamente triunfará una filosofía que se arroje en brazos de lo prohibido puesto que "hasta hoy lo que se ha venido prohibiendo sistemáticamente ha sido la verdad". La educación en la escuela apenas ha influido sobre la capacidad de los estudiantes para aclarar el sentido de su vida. 
De esta manera, el referido autor afirma que más allá de los contenidos teóricos necesarios para la formación en conocimientos de los niños y jóvenes en las escuelas, se debe reflexionar en relación con los valores que se están inculcando, porque, al fin y al cabo, son esos los forjadores de su futuro, que los guiarán como hombres y mujeres del mañana, y a quienes necesariamente desde temprana edad, estamos obligados a orientar por los senderos más positivos y correctos, para que se les permita encontrar sentido a sus vidas y respuestas a sus inquietudes, lo que les favorecerá alcanzar la felicidad de vivir en sociedad, con libertad y justicia.

\section{Conclusiones}

En las páginas anteriores, se ha defendido que la autonomía humana es una, aunque compleja, no se niega que la autonomía es la capacidad que tiene la persona de tomar decisiones por sí mismo, en función de las experiencias y significados construidos a lo largo de todo su vida, al fortalecerla estaríamos potenciando el yo interno, el sí mismo, de cada persona y estaríamos cada vez más, lejos de construir a lo largo de toda nuestra existencia un proceso heterónimo cada vez más arraigado en nosotros, donde la voluntad de cada uno, estaría determinada no por la razón del sujeto, sino por algo ajeno a ella (la voluntad de otras personas, las cosas del mundo, la sensibilidad, la voluntad divina) determinaciones ajenas a sí mismo, que niega y limita en todo momento las circunstancias en que se forma un ser independiente, capaz de darse a sí misma sus propias leyes. A partir de esta reflexión se derivan las siguientes conclusiones:

- La autonomía es inherente a cada persona, que se desarrolla o atrofia dependiendo de las experiencias vividas por la persona a lo largo de toda la vida.

- El reconocimiento de la capacidad de nuestros estudiantes de expresar su voluntad y su razón desde su propio interior debe llevarnos como formadores a aceptar que nos debemos revisar continuamente para reconocer que hemos tenido y mantenemos en nuestros hombros el peso del desarrollo de la autonomía o la formación de cada día más estudiantes y egresados universitarios con marcados conceptos de moral heteronomíca

- El reconocimiento a nuestras prácticas y estilos de enseñar de manera perversa, no debe llevarnos a soslayar nuestra labor como formadores de formadores, que tenemos en nuestras manos desafíos que enfrentan los sistemas educativos en todo el mundo: 
formar personas que tengan actitudes éticas, tanto en la vida privada, como en la vida pública. De nuevo la labor profesorado es en este aspecto fundamental, en tanto, como vimos, la identidad no puede ser algo aprendido, sino el fruto de una indagación que se gesta y madura en el proceso de aprendizaje que es también un proceso de investigación, de indagación permanente. Toda acción educativa es posible siempre y cuando se asuma de acuerdo a los nuevos sujetos, las nuevas realidades.

- La universidad como institución de educación, no puede dejar de hacerse cargo, a partir de la noción de identidad que aquí hemos esbozado, de la parte que le corresponde en este proceso de gestación de identidades. Pareciera ineludible, pues, poner sobre la mesa las condiciones materiales de existencia de los sujetos y los cambios que en las mismas se han producido en los últimos tiempos. Una afirmación como ésta podría parecer descabellada si pensamos en la magnitud de los problemas de orden político, económico y social que afecta no sólo a los países aisladamente considerados sino también en el contexto internacional.

- Ahora bien, el hecho de que existan, es más, predominen las relaciones de dominación no debe desalentarnos en el intento de perseguir como ideal regulativo un modelo de educación integral que permita la formación de sujetos reflexivos y autocríticos. Y no existe otra manera más que asumiéndonos como miembros de una comunidad, que es la que moldea en gran parte nuestro modo de concebirnos, y lo que es más, conscientes de que la propia comunidad a la que pertenecemos se reconoce a sí misma, fija sus límites u horizontes normativos en el encuentro constante con las otras comunidades. Necesitamos de los otros, para la definición de nuestra propia identidad no sólo como personas, sino también como comunidad, como pueblo, como institución, incluso. En este ámbito de creación de espacios comunes de encuentro, diálogo y reconocimiento, la universidad, según la entiendo, tiene un gran reto que afrontar. 


\section{Referencias}

Alanis, H (2003). El saber hacer en la profesión docente. México: Trillas.

Barriga A., Frida y Hernández, R (1998). Estrategias docentes para un aprendizaje significativo. México: McGraw-Hill.

Berner, R (1988). Mandato moral o autonomía. Barcelona: Laia

De Luca, C. (2008) Psicología Evolutiva. Fondo Editorial. Unellez. Barinas. Venezuela

De Zubiría, J. (2005). ¿Qué modelo pedagógico subyace a su práctica educativa? Revista Magisterio Educación y Pedagogía . No 12.Bogota: CREDI.

Díaz B, \& Hernández, R. (2007).Estrategias Docentes para un Aprendizaje Significativo. México: Mc Graw Hill.

Freire, P (1997).Contribuciones para la pedagogía. Buenos Aires: FLACSO

García, F. (1999).Principios y parámetros modernizadores del currículum. Venezuela: UCLA.

González, M. (1997). La motivación académica. Sus determinantes y pautas de intervención. La innovación educativa. Madrid: $\underline{\text { Akal. }}$.

González, M. (1997). La motivación académica. Sus determinantes y pautas de intervención. La innovación educativa. Madrid: Akal.

Larousse (1995).Diccionario. Barcelona: Larousse.

Masiá, J. (1992). Revisión de la heteronomía en diálogo con P. Ricoeur. Isegoría, 5, 17-27.

Masiál, C. (2004). Revisión de la heteronomía en diálogo con P. Ricoeur. Isagoria. Revista de filosofía moral y política. Madrid. España.

Matheus, B. (2002).Economía de la investigación. Proyecto de tesis doctoral. Caracas: USR, LINEA-I.

Pérez A. (1994).Comprender y transformar. La enseñanza. Madrid: Morata.

Riso, W (2004). Amar o depender. Madrid: Norma.

Rodríguez, M. (1995). Exigencias formativas y alternativas en la formación del profesorado. Revista Ínteruniversitaria de formación del profesorado. Valladolid. España.

Rodríguez, M. (2008). Hacia una didáctica critica. Madrid: Muralla.

Rodríguez, M. (2007). Estrategias de enseñanza. Madrid: Muralla.

Rubio, M. (1992). Contratos de aprendizaje. Universidad de México: México.

Schon, S. (1992). El práctico reflexivo. Cómo piensan los profesionales en la acción. Barcelona: Paidós. 
Serrano, H. (2002). El aprendizaje autónomo: Los contratos de trabajo. Revista Innovación Educativa. Madrid: Akal.

Sevillano, M. y Martín, F. (2003). Estrategias de enseñanza-aprendizaje en la formación del profesorado. Madrid: UNED.

Vadillo, M. (2004). La disonancia cognitiva, o cómo el ser humano se convierte en esclavo de sí mismo. http://www.psicoteca.com

Villar, I. y Vicente, P. (1994) Los procesos reflexivos de los profesores. Universidad de Sevilla. Madrid: Servicios de Publicaciones. 